\title{
Study on the Performances of PLLA/CaSiO3 Composites
}

\author{
Qiuhe Zhang ${ }^{1}$, Lisha Zhao ${ }^{1,2}$, Ting Deng ${ }^{1}$ and Yanhua Cai ${ }^{1,2 *}$ \\ ${ }^{1}$ Chongqing Key Laboratory of Environmental Materials \& Remediation Technologies, Chongqing University of Arts and Sciences, \\ Chongqing-402160, P.R. China \\ ${ }^{2}$ College of Chemistry and Environmental Engineering, Chongqing University of Arts and Sciences, Chongqing-402160, P.R. China
}

\begin{abstract}
In the current work, calcium silicate (CaSiO3) was blended with the poly (L-lactic acid) (PLLA) to prepare the PLLA/CaSiO3 composites, and the crystallization process, melting process and thermal decomposition behavior of PLLA/CaSiO3 composites were studied. The promoting effect of $\mathrm{CaSiO} 3$ on the crystallization of PLLA was proved by the melt-crystallization, and the $\mathrm{CaSiO} 3$ content was a crucial factor to the crystallization behavior of PLLA. The crystallization temperature significantly affected the melting behavior of PLLA, and the double melting peaks degenerated into the single melting peak with the increasing of crystallization temperature from $90{ }^{\circ} \mathrm{C}$ to $130{ }^{\circ} \mathrm{C}$. Additionally, for a given PLLA/CaSiO3 sample, the different melting processes in heating after the melt-crystallization results from the different heating rates, and the appearance of the cold-crystallization peak indicated the medium nucleation capacity of CaSiO3. The presence of $\mathrm{CaSiO} 3$ decreased the onset thermal decomposition temperature of PLLA, and this effect depended on the competitive relationship of the poor compatibility of PLLA with CaSiO3 and the content of $\mathrm{CaSiO} 3$ with high thermal stability.
\end{abstract}

\section{Introduction}

Calcium silicate (CaSiO3) has been large-scale application in traditional industries such as construction materials, insulation materials and refractory because of its unique physical and chemical properties and mineralogical characteristics [1]. In recent years, according to the excellent biological activity of $\mathrm{CaSiO} 3$, the $\mathrm{CaSiO} 3$ has attracted increasing attentions in fields of drug delivery and bone tissue engineering [2-4]. For example, Zhang et al employed 3D gel printing technology to prepare the porous $\mathrm{CaSiO} 3$ scaffolds for bone tissue engineering, and the prepared $\mathrm{CaSiO} 3$ power had the average particle size of about $5.119 \mu \mathrm{m}$. After sintering, the scaffolds had well-distributed designed voids and microporous with $62 \%$ porosity, and the compressive strength is up to about $16.52 \mathrm{MPa}$ [5]. Additionally, on the basis of development of more indepth works, $\mathrm{CaSiO} 3$ with different morphologies of nanowires, nanobelts and hollow microspheres was successfully prepared using some methods such as solidstate synthesis, sol-gel method and chemical precipitation method [1], which promotes the $\mathrm{CaSiO} 3$ to be used in more fields.

Poly(L-lactic acid) (PLLA) is a typical biodegradable polymer which has been applied in packaging [6], antibacterial materials [7], drug release [8] and agriculture [9] due to its inherent excellent performances like renewability, biodegradability, easy process, etc [10]. However, the PLLA also suffers from some defects of low glass transition temperature, poor heat resistance, slow crystallization rate and low crystallinity [11]. To overcome slow crystallization speed of PLLA, many additives were selected or prepared to accelerate PLLA's crystallization, these typical additives for improving PLLA's crystallization were talc [12], TMC [13], inorganic salts [14]. For instance, Zhao et al synthesized $\mathrm{N}, \mathrm{N}^{\prime}$-sebacic bis (hydrocinnamic acid) dihydrazide to accelerate the crystallization of PLLA, and the $1 \mathrm{wt} \% \mathrm{~N}$, $\mathrm{N}^{\prime}$-sebacic bis (hydrocinnamic acid) dihydrazide could make the crystallization temperature increase to $131.6^{\circ} \mathrm{C}$, and the nucleation mechanism was proposed to be chemical nucleation [15].

In the current work, $\mathrm{CaSiO} 3$ was added into PLLA resin to prepare the PLLA/CaSiO3 composites, and the performances of melt-crystallization, melting behavior and thermal decomposition of PLLA/CaSiO3 composites were studied via the relevant testing instruments, and this work may be beneficial to develop more bio-based polymer composites.

\section{Experimental section}

\subsection{Materials}

PLLA in this study was produced by Nature Works LLC of USA, and the $\mathrm{CaSiO} 3$ was purchased from Chongqing Huanwei Chemical Company of China.

\footnotetext{
*E-mail: caiyh651@aliyun.com
} 


\subsection{Preparation of PLLA/CaCO3}

The dried PLLA and $\mathrm{CaSiO} 3$ were firstly mixed according to the set mass ratio of PLLA and $\mathrm{CaSiO} 3$ (99.7/0.3, $99.5 / 0.5,99 / 1,98 / 2,97 / 3,95 / 5)$, and then the mixture was melted on a torque rheometer to promote the $\mathrm{CaSiO} 3$ to be further dispersed in PLLA matrix, and the melting temperature was set at $190{ }^{\circ} \mathrm{C}$ during blend, the rotation rate was $32 \mathrm{rpm}$. Finally, the mixture was hot pressed and cool pressed to form the resulting PLLA/CaSiO3 composites.

\subsection{Test}

The melt-crystallization and melt processes of PLLA/CaSiO3 composites were recorded by Q2000 DSC, and testing conditions were $5 \sim 7 \mathrm{mg}$ testing sample, 50 $\mathrm{ml} / \mathrm{min}$ nitrogen and indium standard calibration before testing. The thermal decomposition process curves of PLLA/CaSiO3 composites were obtained by Q500 TGA under flowing air.

\section{Results and discussion}

\subsection{Melt-crystallization}

Figure.1 is the melt-crystallization process DSC curves of the pure PLLA and PLLA/CaSiO3 composites at a cooling rate of $1{ }^{\circ} \mathrm{C} / \mathrm{min}$. As reported, the DSC curve of the pure PLLA can almost not observe any peak, meaning that the crystals cannot form in cooling. Although the presence of $\mathrm{CaSiO} 3$ leads to the appearance of the meltcrystallization peak, the effect of the $\mathrm{CaSiO} 3$ 's content on the melt-crystallization process of PLLA is irregular as shown in Fig. 1. When the CaSiO3 is $0.3 \mathrm{wt} \% \sim 1 \mathrm{wt} \%$, the $\mathrm{CaSiO} 3$ exhibits a negative influence on the crystallization process of PLLA, that is to say, the crystallization occurs at the lower temperature with an increase of $\mathrm{CaSiO} 3$ content. In particular, when the $\mathrm{CaSiO} 3$ is $1 \mathrm{wt} \%$, only few crystals are formed in DSC curves, showing the $1 \mathrm{wt} \% \mathrm{CaSiO} 3$ has the minimum promoting effect for PLLA's crystallization. Whereas the crystallization occurs at the higher temperature when increasing $\mathrm{CaSiO} 3$ content from $1 \mathrm{wt} \%$ to $5 \mathrm{wt} \%$, and the PLLA $/ 5 \% \mathrm{CaSiO} 3$ sample has the sharpest meltcrystallization peak located in the highest temperature comparing with the other PLLA/CaSiO3 sample, indicating that $5 \mathrm{wt} \% \mathrm{CaSiO} 3$ is the optimum concentration in this study. Overall, the addition of $\mathrm{CaSiO} 3$ promotes the melt-crystallization of PLLA, because the $\mathrm{CaSiO} 3$ as a heterogeneous nucleus plays an important role in increasing the nucleation speed in PLLA composites; but the irregular variation of crystallization behavior with increasing of $\mathrm{CaSiO} 3$ content displays the complex crystallization process.

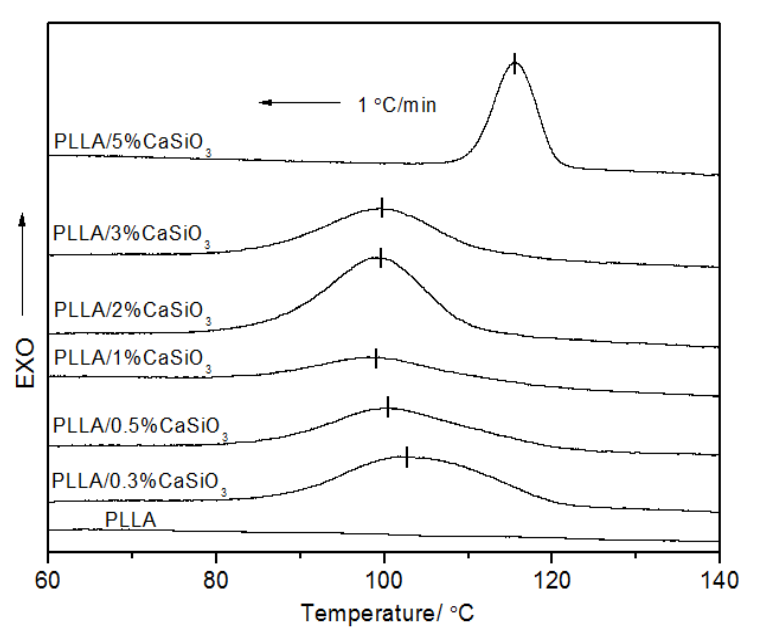

Fig 1. Melt-crystallization process of the pure PLLA and PLLA/CaSiO3 at $1{ }^{\circ} \mathrm{C} / \mathrm{min}$

\subsection{Melting process}

Figure. 2 is the melting process of $\mathrm{PLLA} / 2 \% \mathrm{CaSiO} 3$ sample after crystallization at different temperatures for $180 \mathrm{~min}$. It is observed that, with increasing of crystallization temperature, the low temperature melting peak shifts to the higher temperature, and the high temperature melting peak moves toward the low temperature. As a result, the double melting peaks gradually degenerated into the single melting peak. This result showed that the melting behavior of PLLA/CaSiO3 samples significantly depends on the crystallization temperature, in addition, although that the crystallization time is very enough, the crystallization in the low temperature region cannot still be completed, resulting in that the recrystallization behavior occurs in heating. Furthermore, the lower the crystallization temperature is, the more the number of crystals reformed in heating is. When the crystallization temperature is $130{ }^{\circ} \mathrm{C}$, the $\mathrm{PLLA} / 2 \% \mathrm{CaSiO} 3$ sample only exhibits the single melting peak, the reason is that, in the high temperature region of $130{ }^{\circ} \mathrm{C}$, the mobility of PLLA molecular segment is very high, leading to the difficulty in reforming the crystals.

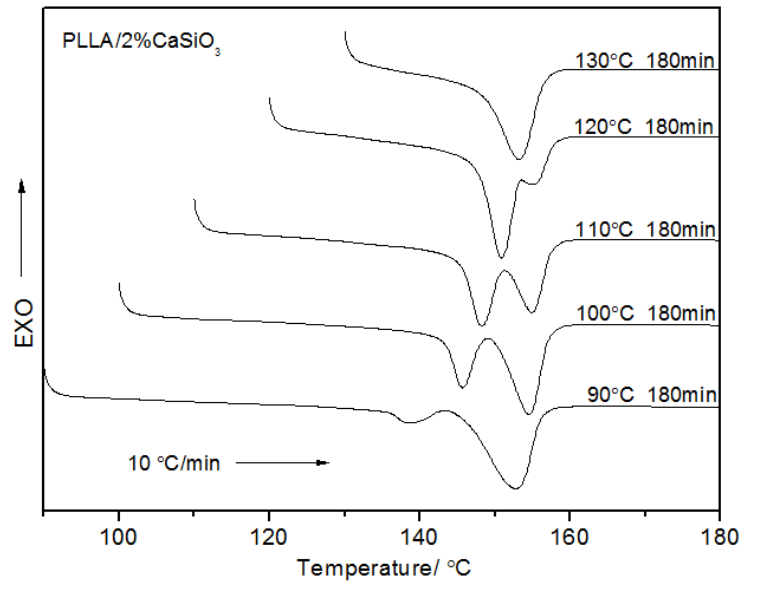

Fig 2. Melting process of PLLA $/ 2 \% \mathrm{CaSiO} 3$ at a heating rate of $10^{\circ} \mathrm{C} / \mathrm{min}$ after crystallization

Additionally, the melting process of PLLA $/ 2 \% \mathrm{CaSiO} 3$ sample at different heating rates after 
cooling of $1{ }^{\circ} \mathrm{C} / \mathrm{min}$ was further investigated by DSC. The low temperature melting peak becomes more obvious with increasing of the heating rate, and the peak area ratio of high temperature melting peak and low temperature melting peak also becomes smaller. It is noted from Fig.3 that the cold-crystallization significantly occurs, when the heating rate is $3{ }^{\circ} \mathrm{C} / \mathrm{min}$ and $5^{\circ} \mathrm{C} / \mathrm{min}$. This result implied that the crystallization cannot be completed after cooling of $1{ }^{\circ} \mathrm{C} / \mathrm{min}$. Meantime, when the heating rate is lower than $5{ }^{\circ} \mathrm{C} / \mathrm{min}$, the high temperature melting peak shifts toward the low temperature with increasing of the heating rate, the reason is that the high temperature melting peak results from the melt of the reformed crystals in heating, and the slower heating rate can provide more time to form the more prefect crystals in heating. However, an enough fast heating rate can make the melting peak appear in the high temperature region because of the thermal inertia.

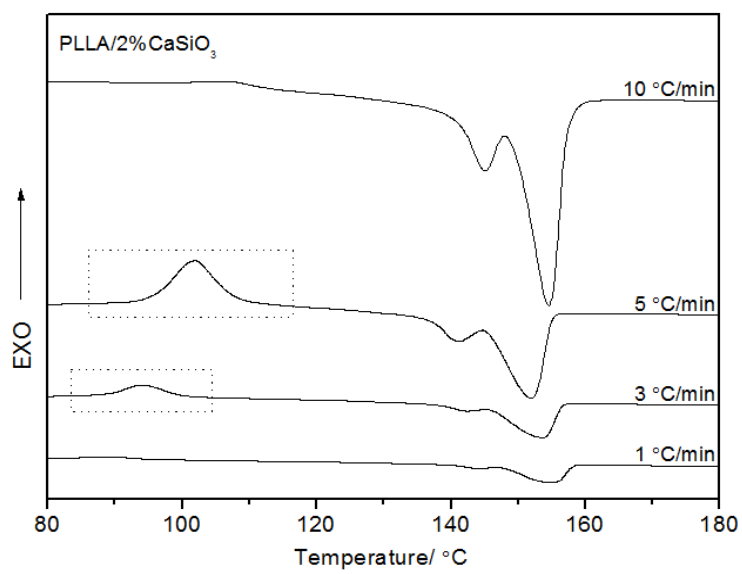

Fig 3. DSC curves of PLLA $/ 2 \% \mathrm{CaSiO} 3$ sample at different heating rates after melt-crystallization

\subsection{Thermal decomposition}

Figure. 4 is the TGA curves of the pure PLLA and PLLA/CaSiO3 samples at a heating rate of $5{ }^{\circ} \mathrm{C} / \mathrm{min}$. As shown in Fig.4, a higher $\mathrm{CaSiO} 3$ loading can cause the onset decomposition temperature (Tod) to decrease in the concentration range of $0.3 \mathrm{wt} \%$ to $2 \mathrm{wt} \%$, because the compatibility of PLLA with $\mathrm{CaSiO} 3$ is very poor, which results in the more defect of PLLA/CaSiO3 composites, and these defects can be firstly destroyed in heating. However, further increasing the $\mathrm{CaSiO} 3$ content can elevate the Tod, resulting from the high decomposition temperature of $\mathrm{CaSiO} 3$. In a word, the Tod depends on the negative effect of poor compatibility of PLLA with $\mathrm{CaSiO} 3$ and positive effect of $\mathrm{CaSiO} 3$ with high decomposition temperature. Additionally, the only one thermal decomposition stage in TGA curve shows that the $\mathrm{CaSiO} 3$ content in this study cannot change the thermal decomposition profile of PLLA.

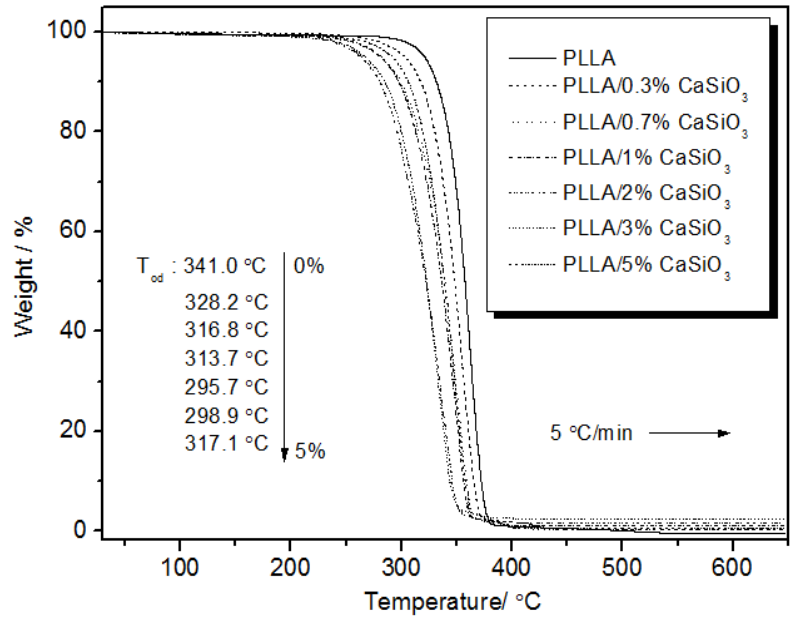

Fig 4. Thermal decomposition of the pure PLLA and PLLA/CaSiO3 samples

\section{Conclusions}

PLLA/CaSiO3 composites was prepared via the melting blend technology, and the thermal properties of the composites were investigated by DSC and TGA. CaSiO3 as a heterogeneous nucleus could accelerate the crystallization of PLLA, and $5 \mathrm{wt} \% \mathrm{CaSiO} 3$ exhibited the best promoting effect for PLLA's crystallization, but the crystallization process was complex. Both crystallization temperature and heating rate could significantly affected the melting processes of PLLA/CaSiO 3 composites, and the thermal stability of the composites was decreased due to the introduction of $\mathrm{CaSiO} 3$.

\section{Acknowledgments}

This work was supported by Foundation of Chongqing Municipal Science and Technology Commission (cstc2017shmsA20021 and cstc2019jcyj-msxmX0876), Scientific and Technological Research Program of Chongqing Municipal Education Commission (project number KJQN201801319).

\section{References}

1. Wang, Y., Guo, X.H., Zhou, G.D., Cheng, T.X. (2019) Effect of alkyl benzene sulfonate surfactant on morphology and structure of calcium silicate hydrate. Chem. J. Chinese., 40: 1795-1804.

2. Zhang, C.M., Wei, W., Zhang, J.Y., Li, Y.S., Zhou, G.Q., Jia, G. (2017) Uniform mesoporous CaSiO3:Eu3+ nanospheres: Template-directed synthesis, luminescence and sustained drug release properties. Dyes. Pigments., 136: 427-433.

3. Zhao, L., Wu, C.T., Lin, K.L., Chang, J. (2012) The effect of poly(lactic-co-glycolic acid) (PLGA) coating on the mechanical, biodegradable, bioactive properties and drug release of porous calcium silicate scaffolds. Bio-med. Mater. Eng., 22: 289-300. 
4. Ni, S.Y., Chang, J., Chou, L. (2006) A novel bioactive porous $\mathrm{CaSiO} 3$ scaffold for bone tissue engineering. J. Biomed. Mater. Res. A, 76: 196-205.

5. Zhang, Z.N., Shao, H.P., Lin, T., Zhang, Y.M., He, J.Z., Wang, L.H. (2019) 3D gel printing of porous calcium silicate scaffold for bone tissue engineering. J. Mater. Sci., 54: 10430-10436.

6. Genovese, L., Soccio, M., Lotti, N., Gazzano, M., Siracusa, V., Salatelli, E., Balestra, F., Munari, A. (2017) Design of biobased PLLA triblock copolymers for sustainable food packaging: Thermomechanical properties, gas barrier ability and compostability. Eur. Polym. J., 95: 289-303.

7. Zhou, Y.W., Hu, K., Guo, Z.B., Fang, K., Wang, X., Yang, F., Gu, N. (2017) PLLA microcapsules combined with silver nanoparticles and chlorhexidine acetate showing improved antibacterial effect. Mater. Sci. Eng. C., 78: 349-353.

8. Ding, A.M., Teng, L.J., Zhou, Y.F., Chen, P.P., Nie, W.Y. (2018) Synthesis and characterization of bovine serum albumin-loaded microspheres based on starshaped PLLA with a xylitol core and their drug release behaviors. Polym. Bull., 75: 2917-2931.

9. Rocha, D.B., Souza de Carvalho, J., de Oliveira, S.A., dos Santos Rosa, D. (2018) A new approach for flexible PBAT/PLA/CaCO3 films into agriculture. J. Appl. Polym. Sci., 135: 46660.

10. Ma, M., Xu, L., Liu, K., Chen, S., He, H.W., Shi, Y.Q., Wang, X. (2020) Effect of triphenyl phosphite as a reactive compatibilizer on the properties of poly(L-lactic acid)/poly(butylene succinate) blends. J. Appl. Polym. Sci., 137): 48646.

11. Sun, J.R., Yu, H.Y., Zhuang, X.L., Chen, X.S., Jing, X.B. (2011) Crystallization behavior of asymmetric PLLA/PDLA blends. J. Phys. Chem. B., 115: 28642869.

12. Li, Y., Han, C.Y., Yu, Y.C., Xiao, L.G., Shao, Y. (2019) Effect of content and particle size of talc on nonisothermal melt crystallization behavior of poly(L-lactide). J. Therm. Anal. Calorim., 135: 20492058.

13. Ding, S.F., Luo, F.L., Wang, K.Z., Xing, Q., Si, P.F., Lei, X.M., Shen, Z.Y. (2016) Influence of nucleator TMC-210 on crystallization and properties of PLLA. Polym. Mater. Sci. Eng., 32: 66-72.

14. Han, L.J., Han, C.Y., Bian, J.J., Bian, Y.J., Lin, H.J., Wang, X.M., Zhang, H.L., Dong, L.S.(2012) Preparation and characteristics of a novel nano-sized calcium carbonate (nano-CaCO3)-supported nucleating agent of poly(L-lactide). Polym. Eng. Sci., 52: 1474-1484.

15. Zhao, L.S., Cai, Y.H., Liu, H.L. (2019) N, N'-sebacic bis(hydrocinnamic acid) dihydrazide: A crystallization accelerator for poly(L-lactic acid). EPolymers, 19: 141-153 